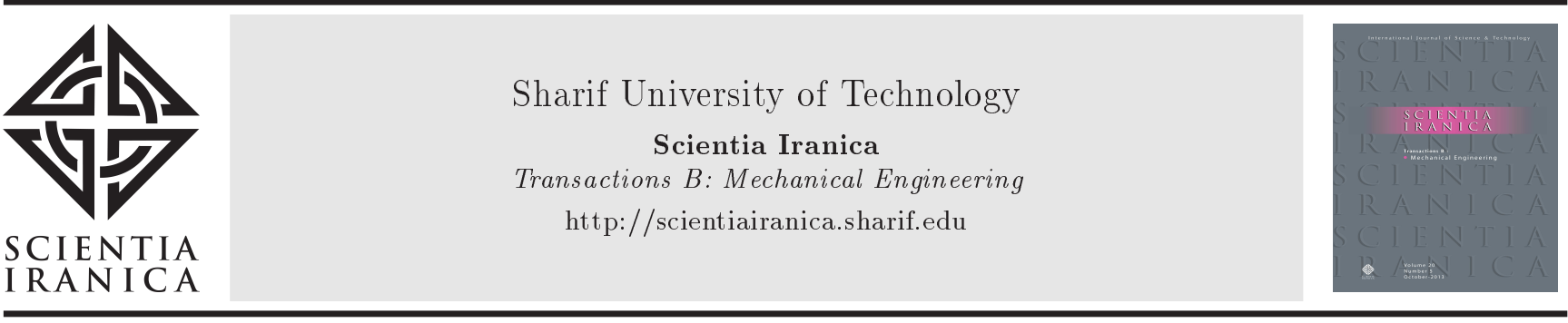

Research Note

\title{
Investigation into size effect on lateral vibrations of a micro-drill subjected to an axial load using the modified couple stress theory
}

\author{
A. Rahi* \\ Faculty of Mechanical and Energy Engineering, Shahid Beheshti University, Tehran, Iran.
}

Received 6 August 2017; received in revised form 5 April 2018; accepted 14 May 2018

\author{
KEYWORDS \\ Micro-drill; \\ Modified couple stress \\ theory; \\ Size-dependency; \\ Free vibration; \\ Axial load.
}

\begin{abstract}
In this paper, the modified couple stress theory is used to capture size effect on dynamic behavior of a micro-drill subjected to an axial load and a concentrated mass, which is attached to its free end. Governing equations of lateral vibrations of the system and associated boundary conditions were derived by first, obtaining the total kinetic and potential energy of the system and then, using Hamilton's principle. The Assumed Modes method was applied for transforming the governing partial differential equations into a set of infinite ordinary differential equations. Considering two terms of the equations, the first two natural frequencies and instability rotational speeds of the micro-drill system were determined semi-analytically. Finally, numerical results of natural frequencies and the threshold of instability speeds of the system were derived with respect to different values of the system parameters such as rotational speed, axial load, rotor length, concentrated mass, and material length scale. The results showed that the material length scale parameter was extremely effective on natural frequencies and the threshold of instability speeds of the micro-drill.

(C) 2019 Sharif University of Technology. All rights reserved.
\end{abstract}

\section{Introduction}

Recently, with the development of new technologies for manufacturing devices at small sizes (e.g., micron or sub-micron scales), manufacturing of many smallsize components for devices in the field of the MicroElectro-Mechanical Systems (MEMS) has been possible. For example, micro-engines and micro-drills are two types of micro-rotating equipment, which can be used in industrial or biomedical applications [16]. Generally, the rotational speed of the rotor in micro-drills is very high and can reach $60000 \mathrm{rpm}$ [6]. Therefore, determining the lateral vibrations behavior of the micro-rotating machines is of significance.

\footnotetext{
*.E-mail address: a_rahi@sbu.ac.ir
}

Some researchers investigated dynamic response of the micro-rotating equipment in the field of MEMS based on the classical continuum mechanics theory $[7,8]$. It should be noted that capturing the size effect is not possible using the classical continuum mechanics theories. Considering the size effect is a significant challenge in the study of mechanical behavior of the micro-structures. The size dependency of mechanical behavior of the materials in small sizes has been observed by experimental methods for metals and polymers [9-13]. The classical continuum mechanics theories are unable to explain and predict the mechanical behavior of the materials in small sizes. Therefore, some of the non-classical continuum theories such as strain gradient theory, couple stress theory, nonlocal elasticity theory, and surface elasticity theory have been introduced to capture the size effect on elastic deformation behavior of micro-structures [14-23]. 
In some non-classical continuum theories such as couple stress theory, two material length scale parameters are used to describe the size effect on the mechanical behavior of materials. Yang et al. [16] proposed the Modified Couple Stress Theory (MCST) to explain size dependency by using only one material length scale parameter. They introduced the effect of the moment of the couple in addition to the classical equations of forces in the modified couple stress theory.

Again, Mindlin [24] and Mindlin and Eshel [25] presented another form of higher-order continuum theory and a general form of strain gradient elasticity theory to consider the size effect by using first and second gradients of the strain tensor in the strain energy density. By considering only the first gradient of the strain tensor, Fleck and Hutchinson [26] reformulated and modified Mindlin's suggested theory and named it Strain Gradient Theory (SGT). The strain gradient theory had more terms of higher-order stress components than the couple stress theory. Afterwards, Lam et al. [27] proposed the Modified Strain Gradient Theory (MSGT) to predict the size effect on the mechanical behavior of materials by using only three constants of material length scale parameters.

The MCST has been employed by several researchers to study the size dependency of the static and dynamic mechanical behaviors of materials in small size. For example, the modified couple stress theory has been used as a basic formulation to study sizedependent effect of static and dynamic behaviors of linear and nonlinear micro-beams [28-35], vibration response of rotating FGM micro-beams [36], static and dynamic behaviors of rectangular and annular sectors of microplates [37-45], curved micro-tubes [46], and micro/nano spherical shells [47].

Vatankhah and Kahrobaiyan [48] studied vibration of a micro-resonator based on MSGT to capture size effect on the system. They modeled the microresonator as a clamped-clamped micro-beam subjected to a tensile axial force with a concentrated mass attached to the micro-beam. Lead-lag vibration characteristics of a rotating micro-cantilever beam based on MCST and both Euler-Bernoulli and Timoshenko beam theories were studied by Dehrouyeh-Semnani et al. [49]. They used the finite element method to calculate natural frequencies of the system. Hashemi and Asghari [50] investigated lateral vibrations of an elastic micro-rotating shaft-disk system by considering small-scale effects based on MCST. They considered a disk attached to the middle of a flexible microrotating shaft and then, by using the Galerkin approach, obtained the first two natural frequencies of the system with respect to different values of size scale parameter. In addition, there are several studies of the dynamic response of overhung rotor systems based on the classical continuum mechanics theories $[51,52]$.
According to the above literature review, there are no reported investigations into lateral vibrations behavior of a micro-cantilever rotor system subjected to an axial load with a concentrated mass attached to its free end, called micro-drill system, to consider its size dependency.

In the present article, for a real dental drill, which is used in the medical industry, a micro-drill system is modeled into a micro-cantilever rotor subjected to an axial load with a concentrated mass attached to its free end. It can be used in the future applications in medical industries. Then, the modified couple stress theory is used to capture small-size effects on lateral vibrations behavior of the micro-drill rotor system. After derivation of kinetic and potential energy of the system, the governing equations of motion and associated boundary conditions are obtained using Hamilton's principle based on MCST. Then, to reach semi-analytical results for lateral vibrations characteristics, the Assumed Modes method is employed to transform the governing equations into a set of infinite ordinary differential equations. The first and second natural frequencies for both forward and backward whirling motions of the system and rotational speed values, which cause instability, are obtained. Finally, numerical results of the first two natural frequencies and instability rotational speeds of the micro-drill rotor are presented with respect to different values of the system parameters, such as the material length scale, rotational speed, axial load, rotor length, and concentrated mass, which is attached to the free end of the micro-cantilever rotor.

\section{Theory and mathematical equations}

\subsection{Modeling}

Figure 1 shows a real dental or bone drill, which is used in medical industries. In recent years, with the growth of new technologies for manufacturing devices at small sizes (e.g., micron scale), production of many components with small sizes in the field of MicroElectro-Mechanical Systems (MEMS) is possible. Also, the rotational speed of the rotor in a micro-drill is very high and can reach $60000 \mathrm{rpm}$ [6].

In this study, the real dental drills in macro sizes are applied for modeling of micro-scale drills. The idea of micro-drills can be utilized in future applications in medical industries.

The micro-drill system is modeled as a microcantilever rotor with a concentrated mass attached to the free end of the rotor subjected to an axial load, as shown in Figure 2.

The flexible micro-rotor, which rotates at a constant rotational speed $\Omega$, has the length $L$, radius $R$, density $\rho$, and circular cross section area $A$. Also, the coordinate system $X-Y-Z$, constant axial load $P$, 


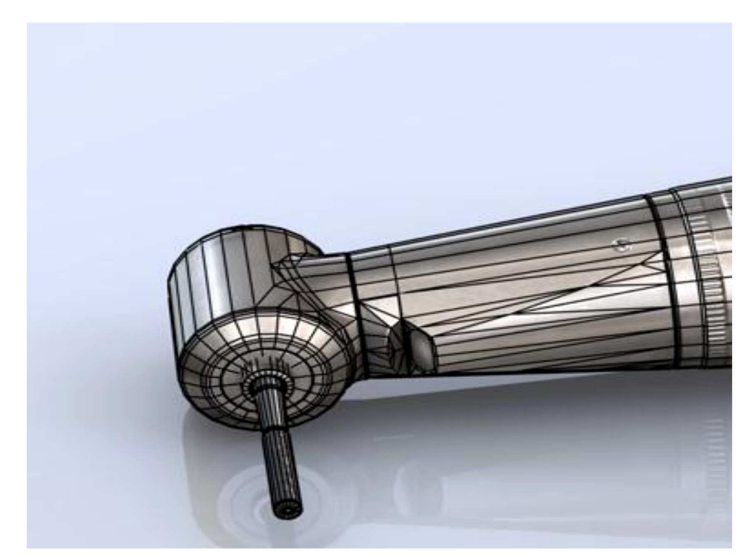

(a)

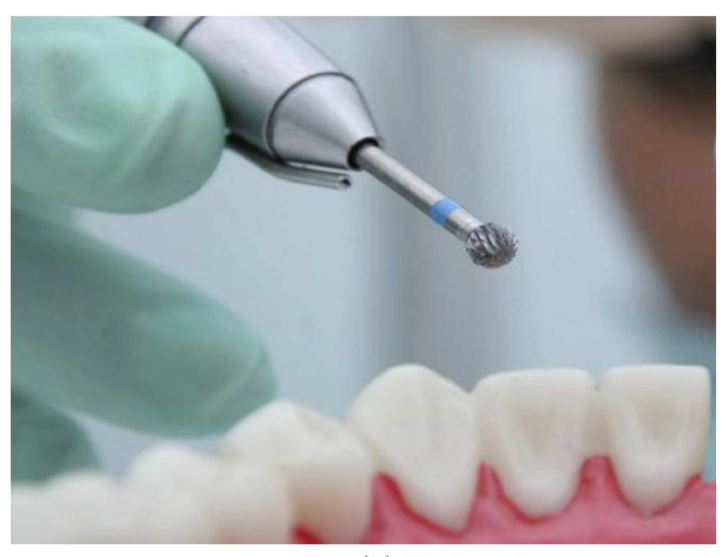

(b)

Figure 1. Real pictures of a dental drill used in medical industries: (a) and (b) respectively, without [53], and with [54] concentrated mass at the free end of the rotor.

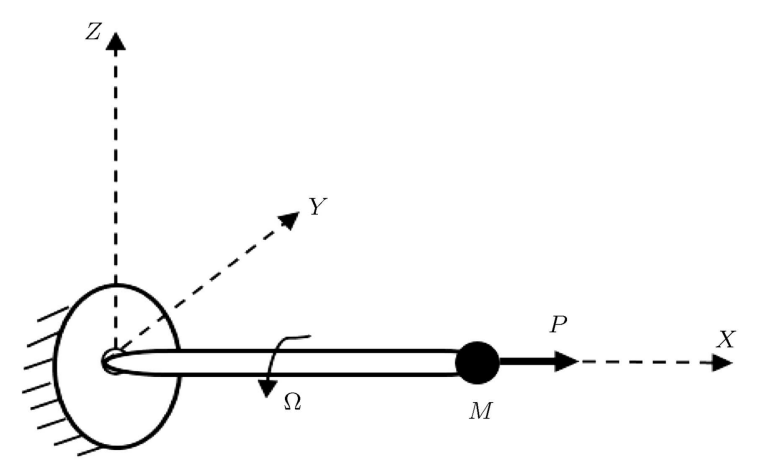

Figure 2. Modelling of a micro-drill system into a micro-cantilever rotor with a concentrated mass, $M$, attached to free end of the rotor subjected to an axial load $P$.

and concentrated mass (point mass) $M$ are considered, as represented in Figure 2.

\subsection{The modified couple stress theory}

The strain energy density of a linear elastic isotropic material based on the modified couple stress theory can be written as follows [16,28]:

$$
\pi_{0}=\frac{1}{2} \sigma_{i j} \varepsilon_{i j}+\frac{1}{2} m_{i j} \chi_{i j}^{s}
$$

where the corresponding work-conjugated stress can be expressed as follows:

$$
\begin{aligned}
& \sigma_{i j}=2 \mu \varepsilon_{i j}+\lambda \varepsilon_{k k} \delta_{i j}, \\
& m_{i j}=2 \mu l^{2} \chi_{i j}^{s},
\end{aligned}
$$

where:

$$
\begin{aligned}
& \varepsilon_{i j}=\frac{1}{2}\left(u_{i, j}+u_{j, i}\right), \\
& \chi_{i j}^{s}=\frac{1}{2}\left(\theta_{i, j}+\theta_{j, i}\right), \\
& \theta_{i}=\frac{1}{2} \varepsilon_{i j k} u_{k, j} .
\end{aligned}
$$

In the above equations, $\theta_{i, j}$ denotes gradient of rotation, $\theta_{i}$ denotes infinitesimal rotation vector, $\epsilon_{i j k}$ denotes alternating tensor (or permutation symbol), and $u_{i}$ denotes components of the displacement vector. The parameters $(\lambda, \mu)$ and $l$ are called Lamé constants and the material length scale parameter, respectively. The Lamé constants can also be written regarding Young's modulus, $E$, and Poisson's ratio, $\vartheta$.

\subsection{Governing equations of motion}

According to Figure 2, $u(x, t), v(x, t)$, and $w(x, t)$ denote the longitudinal and lateral deflections of the midline of the micro-elastic rotor at any point $x$ along the length of the rotor in the $X, Y$, and $Z$ directions, respectively. Considering Euler-Bernoulli beam theory, displacement field in the rotor can be expressed as follows [50]:

$$
\begin{aligned}
& u_{1}=u(x, t)-z \frac{\partial w(x, t)}{\partial x}-y \frac{\partial v(x, t)}{\partial x}, \\
& u_{2}=v(x, t) \\
& u_{3}=w(x, t) .
\end{aligned}
$$

Assuming small slope in the rotor after deformation, the non-zero components of strain and stress tensors can be expressed as follows:

$$
\begin{aligned}
& \varepsilon_{x x}=\frac{\partial u_{1}}{\partial x}=\frac{\partial u}{\partial x}-z \frac{\partial^{2} w}{\partial x^{2}}-y \frac{\partial^{2} v}{\partial x^{2}} \\
& \sigma_{x x}=E \varepsilon_{x x}=E\left(\frac{\partial u}{\partial x}-z \frac{\partial^{2} w}{\partial x^{2}}-y \frac{\partial^{2} v}{\partial x^{2}}\right) .
\end{aligned}
$$

It should be noted that in writing Eq. (8), EulerBernoulli beam theory has been used [30]. Also, we have:

$$
\begin{aligned}
& \chi_{13}^{s}=\chi_{31}^{s}=\frac{1}{2} \frac{\partial^{2} v}{\partial x^{2}}, \quad \chi_{12}^{s}=\chi_{21}^{s}=\frac{-1}{2} \frac{\partial^{2} w}{\partial x^{2}}, \\
& \chi_{11}^{s}=\chi_{22}^{s}=\chi_{23}^{s}=\chi_{32}^{s}=\chi_{33}^{s}=0 .
\end{aligned}
$$

Therefore, from Eq. (1), the strain energy density of 
elastic rotor $\pi_{0}$ is given by:

$$
\begin{aligned}
\pi_{0}= & \frac{1}{2} E\left[\left(\frac{\partial u}{\partial x}\right)^{2}+z^{2}\left(\frac{\partial^{2} w}{\partial x^{2}}\right)^{2}+y^{2}\left(\frac{\partial^{2} v}{\partial x^{2}}\right)^{2}\right. \\
& \left.-2 z \frac{\partial u}{\partial x} \frac{\partial^{2} w}{\partial x^{2}}-2 y \frac{\partial u}{\partial x} \frac{\partial^{2} v}{\partial x^{2}}-y z \frac{\partial^{2} v}{\partial x^{2}} \frac{\partial^{2} w}{\partial x^{2}}\right] \\
& +\frac{E l^{2}}{2(1+\vartheta)}\left[\left(\frac{\partial^{2} w}{\partial x^{2}}\right)^{2}+\left(\frac{\partial^{2} v}{\partial x^{2}}\right)^{2}\right] .
\end{aligned}
$$

The strain energy, $\pi_{s}$, of the system, without considering the effect of axial load, $P$, can be determined as follows:

$$
\pi_{s}=\int_{V} \pi_{0} d V=\int_{L}\left[\int_{A} \pi_{0} d A\right] d x
$$

where $V$ denotes volume and $A$ denotes cross section of the elastic rotor at point $x$. By substituting Eq. (10) into Eq. (11), strain energy of the system, without considering the effect of axial load, $P$, can be obtained as follows:

$$
\begin{aligned}
\pi_{s}= & \frac{1}{2} E \int_{0}^{L}\left[A\left(\frac{\partial u}{\partial x}\right)^{2}+I_{y y}\left(\frac{\partial^{2} w}{\partial x^{2}}\right)^{2}+I_{z z}\left(\frac{\partial^{2} v}{\partial x^{2}}\right)^{2}\right] d x \\
& +\frac{E l^{2}}{2(1+\vartheta)} \int_{0}^{L} A\left[\left(\frac{\partial^{2} w}{\partial x^{2}}\right)^{2}+\left(\frac{\partial^{2} v}{\partial x^{2}}\right)^{2}\right] d x
\end{aligned}
$$

where $I_{y y}=\int_{A} z^{2} d A, I_{z z}=\int_{A} y^{2} d A$, and $\int_{A} z d A=$ $\int_{A} y d A=\int_{A} y z d A=0$.

It should be noted that for a uniform and isotropic rotor with circular section radius $R$, the moment of inertia in the cross section area is $I=I_{y y}=I_{z z}=$ $\frac{\pi R^{4}}{4}=\frac{A R^{2}}{4}$. Therefore, Eq. (12) can be written as follows:

$$
\begin{aligned}
\pi_{s}= & \frac{1}{2} E A \int_{0}^{L}\left(\frac{\partial u}{\partial x}\right)^{2} d x+\frac{1}{2} E I \int_{0}^{L}\left[\left(\frac{\partial^{2} w}{\partial x^{2}}\right)^{2}\right. \\
& \left.+\left(\frac{\partial^{2} v}{\partial x^{2}}\right)^{2}\right] d x+\frac{2 E I}{1+\vartheta}\left(\frac{l}{R}\right)^{2} \int_{0}^{L}\left[\left(\frac{\partial^{2} w}{\partial x^{2}}\right)^{2}\right. \\
& \left.+\left(\frac{\partial^{2} v}{\partial x^{2}}\right)^{2}\right] d x .
\end{aligned}
$$

Also, for the axial load $P$, strain energy of the system can be determined as follows:

$$
\pi_{P}=\frac{1}{2} \int_{0}^{L} P(x)\left[2\left(\frac{\partial u}{\partial x}\right)+\left(\frac{\partial w}{\partial x}\right)^{2}+\left(\frac{\partial v}{\partial x}\right)^{2}\right] \underset{(14)}{d x}
$$

where $P(x)$ is the axial load at any point $x$ of the cross section of the micro-drill rotor. It should be noted that in the present paper, $P(x)$ is equal to constant $P$.
Therefore, total strain energy of the system can be obtained as follows:

$$
\begin{aligned}
\pi= & \pi_{s}+\pi_{P}=\frac{1}{2} E A \int_{0}^{L}\left(\frac{\partial u}{\partial x}\right)^{2} d x \\
& +\frac{1}{2} E I \int_{0}^{L}\left[\left(\frac{\partial^{2} w}{\partial x^{2}}\right)^{2}+\left(\frac{\partial^{2} v}{\partial x^{2}}\right)^{2}\right] d x \\
& +\frac{2 E I}{1+\vartheta}\left(\frac{l}{R}\right)^{2} \int_{0}^{L}\left[\left(\frac{\partial^{2} w}{\partial x^{2}}\right)^{2}+\left(\frac{\partial^{2} v}{\partial x^{2}}\right)^{2}\right] d x \\
& +\frac{1}{2} \int_{0}^{L} P\left[2\left(\frac{\partial u}{\partial x}\right)+\left(\frac{\partial w}{\partial x}\right)^{2}+\left(\frac{\partial v}{\partial x}\right)^{2}\right] d x
\end{aligned}
$$

Kinetic energy of the system $T$ can be written as follows:

$$
T=T_{r}+T_{M}
$$

where $T_{r}$ is the kinetic energy of the elastic micro-drill rotor and $T_{M}$ is the kinetic energy of the concentrated mass, $M$, attached to the free end of the microcantilever rotor. Also, we have $[50,55]$ :

$$
\begin{aligned}
& T_{r}=\frac{1}{2} \int_{V} \rho \dot{\vec{r}} \cdot \dot{\vec{r}} d V, \\
& \vec{r}=\left(x+u_{1}\right) \mathbf{i}+\left(y+u_{2}\right) \mathbf{j}+\left(z+u_{3}\right) \mathbf{k},
\end{aligned}
$$

where $\rho, \vec{r}$, and $\dot{\vec{r}}$ are density, position, and velocity vectors of any particle on the micro-elastic rotor, respectively. By simplifying Eq. (17), it can be written as follows $[50,55]$ :

$$
\begin{aligned}
T_{r}= & \frac{\rho}{2} \int_{0}^{L}\left[A\left(\dot{u}^{2}+\dot{v}^{2}+\dot{w}^{2}\right)+I\left(\left(\frac{\partial \dot{w}}{\partial x}\right)^{2}+\left(\frac{\partial \dot{v}}{\partial x}\right)^{2}\right)\right. \\
& \left.+A \Omega^{2}\left(v^{2}+w^{2}\right)+2 A \Omega(v \dot{w}-w \dot{v})\right] d x \\
& +\rho I L \Omega^{2}
\end{aligned}
$$

where the dot over variables is the derivative of variable relative to time.

The kinetic energy of concentrated mass $M$ can also be found as follows [55]:

$$
\begin{aligned}
T_{M}= & \frac{M}{2} \int_{0}^{L} \delta(x-L)\left[\left(\dot{u}^{2}+\dot{v}^{2}+\dot{w}^{2}\right)+\Omega^{2}\left(v^{2}+w^{2}\right)\right. \\
& +2 \Omega(v \dot{w}-w \dot{v})] d x+\frac{I_{M y y}}{2} \int_{0}^{L} \delta \\
& (x-L)\left[\left(\frac{\partial \dot{w}}{\partial x}\right)^{2}+\left(\frac{\partial \dot{v}}{\partial x}\right)^{2}\right] d x+I_{M p} \Omega^{2}
\end{aligned}
$$

where $\delta(x)$ is Dirac delta function and: 


$$
\left(I_{M y y}, I_{M z z}, I_{M p}\right)=\int_{M}\left(z^{2}, y^{2}, y^{2}+z^{2}\right) d M .
$$

It should be noted that for the system with concentrated mass, $I_{M y y}, I_{M z z}$, and $I_{M p}$ are equal to zero (because we neglect the shape of the point mass).

Now, Hamilton's principle is considered as follows:

$$
\int_{t_{1}}^{t_{2}} \delta(T-\pi+W) d t=0
$$

where $\delta T, \delta \pi$, and $\delta W$ are the virtual variation of kinetic energy, the virtual variation of strain energy, and virtual work done by external non-conservative forces on the system, respectively. For the considered system without external forces, $\delta W$ is zero.

By substituting Eqs. (15) and (16) into Eq. (20) and then, using variational calculus, the governing equations of motion of the micro-drill system can be derived as follows:

$$
\begin{gathered}
S \frac{\partial^{4} v}{\partial x^{4}}-P \frac{\partial^{2} v}{\partial x^{2}}+[\rho A+M \delta(x-L)] \\
\left(\ddot{v}-2 \Omega \dot{\mathrm{w}}-\Omega^{2} v\right)-\rho I \frac{\partial^{2} \ddot{v}}{\partial x^{2}}=0, \\
S \frac{\partial^{4} w}{\partial x^{4}}-P \frac{\partial^{2} w}{\partial x^{2}}+[\rho A+M \delta(x-L)] \\
\left(\ddot{w}+2 \Omega \dot{v}-\Omega^{2} w\right)-\rho I \frac{\partial^{2} \ddot{w}}{\partial x^{2}}=0,
\end{gathered}
$$

where:

$$
S=E I\left[1+\frac{2}{1+\vartheta}\left(\frac{l}{R}\right)^{2}\right] .
$$

In the above equations, $R$ is radius of cross section, $\rho$ is density, $\Omega$ is rotational speed of the micro-drill rotor, and $l$ is the material length scale parameter, which is dependent on material property and can be determined by typical experiments such as the micro-bend test.

It should be noted that the terms $[\rho A+$ $M \delta(x-L)]\left(-2 \Omega \dot{\mathrm{w}}-\Omega^{2} v\right)$ and $[\rho A+M \delta(x-L)]$ $\left(2 \Omega \dot{v}-\Omega^{2} w\right)$ in the governing Eqs. (21) and (22) show the rotational speed effect in the micro-drill rotor system. In particular, in case of $\Omega=0$, Eqs. (21) and (22) will be decoupled and they represent the lateral vibrations behavior of the system in $v$ and $w$ directions, respectively, without rotational speed.

Eqs. (21) and (22) represent the nonlinear partial differential equations of lateral motions of the microdrill rotor subjected to an axial load with a concentrated mass based on the modified couple stress theory. Also, the boundary conditions at each end of the microdrill rotor system can be obtained as:

$$
\begin{aligned}
& v(0, t)=w(0, t)=0, \\
& \frac{\partial v}{\partial x}(0, t)=\frac{\partial w}{\partial x}(0, t)=0
\end{aligned}
$$

$$
\begin{aligned}
& \frac{\partial^{2} v}{\partial x^{2}}\left(L^{+}, t\right)=\frac{\partial^{2} w}{\partial x^{2}}\left(L^{+}, t\right)=0, \\
& S \frac{\partial^{3} v}{\partial x^{3}}\left(L^{+}, t\right)-P \frac{\partial v}{\partial x}\left(L^{+}, t\right)=0, \\
& S \frac{\partial^{3} w}{\partial x^{3}}\left(L^{+}, t\right)-P \frac{\partial w}{\partial x}\left(L^{+}, t\right)=0,
\end{aligned}
$$

where $L^{+}$is the length of the micro-drill rotor plus a very small virtual length at the right side of the concentrated mass, $M$. It should be noted that moments of inertia of the concentrated mass, $M$, according to Figure 2, are neglected. Also, in the above governing equations, only the lateral motions of the micro-drill rotor have been considered.

\section{Semi-analytical solution to the governing equations of motion}

In this section, the solutions to Eqs. (21) and (22) with their boundary conditions in Eqs. (24) and (25) are considered. It should be noted that longitudinal motion of the micro-drill rotor system can be neglected besides its lateral motions. To solve the governing equations (Eqs. (21) and (22)), the Assumed Modes method is used. For this purpose, lateral deflections $w(x, t)$ and $v(x, t)$ are considered as follows:

$$
\begin{aligned}
v(x, t) & =\sum_{m=1}^{\infty} \psi_{m}(x) V_{m}(t), w(x, t) \\
& =\sum_{m=1}^{\infty} \psi_{m}(x) W_{m}(t)
\end{aligned}
$$

where $\psi_{m}$ is shape functions that satisfy geometrical boundary conditions of the system, and $V_{m}(t)$ and $W_{m}(t)$ are time-dependent functions, which are called generalized coordinates. Shape functions $\psi_{m}(x)=$ $1-\cos \left(\frac{m \pi x}{2 L}\right)$, with $m=1,2,3, \ldots$, are employed for the micro-cantilever rotor with boundary conditions in Eqs. (24) and (25).

By substituting Eq. (26) into Eqs. (21) and (22), multiplying the results by orthogonal related modes $\psi_{n}(x)$ (with $n$ being an arbitrary natural number), and then taking integration over the rotor length, the following results can be obtained:

$$
\begin{aligned}
\int_{0}^{L} \sum_{m=1}^{\infty} & \left\{\left[S\left(\frac{m \pi}{2 L}\right)^{4} V_{m}+P\left(\frac{m \pi}{2 L}\right)^{2} V_{m}\right.\right. \\
& \left.+\rho I\left(\frac{m \pi}{2 L}\right)^{2} \ddot{V}_{m}\right] \cos \left(\frac{m \pi x}{2 L}\right) \cos \left(\frac{n \pi x}{2 L}\right) \\
& +[\rho A+M \delta(x-L)]
\end{aligned}
$$




$$
\begin{aligned}
& \left(\ddot{V}_{m}-2 \Omega \dot{W}_{m}-\Omega^{2} V_{m}\right) \\
( & \left.\left.1-\cos \left(\frac{m \pi x}{2 L}\right)\right)\left(1-\cos \left(\frac{n \pi x}{2 L}\right)\right)\right\} d x \\
= & 0, \\
\int_{0}^{L} \sum_{m=1}^{\infty} & \left\{\left[S\left(\frac{m \pi}{2 L}\right)^{4} W_{m}+P\left(\frac{m \pi}{2 L}\right)^{2} W_{m}\right.\right. \\
+ & \left.\rho\left(\frac{m \pi}{2 L}\right)^{2} \ddot{W}_{m}\right] \cos \left(\frac{m \pi x}{2 L}\right) \cos \left(\frac{n \pi x}{2 L}\right) \\
& +[\rho A+M \delta(x-L)] \\
& \left(\ddot{W}_{m}+2 \Omega \dot{V}_{m}-\Omega^{2} W_{m}\right) \\
& \left.\left(1-\cos \left(\frac{m \pi x}{2 L}\right)\right)\left(1-\cos \left(\frac{n \pi x}{2 L}\right)\right)\right\} d x \\
& =0 .
\end{aligned}
$$

By calculating the above integrals, the following set of infinite ordinary differential equations can be determined for $n=1,2,3, \ldots$ :

$$
\begin{aligned}
\frac{L}{2}[ & \left.S\left(\frac{n \pi}{2 L}\right)^{4} V_{n}+P\left(\frac{n \pi}{2 L}\right)^{2} V_{n}+\rho I\left(\frac{n \pi}{2 L}\right)^{2} \ddot{V}_{n}\right] \\
& +\sum_{m=1}^{\infty} \rho A L\left[\ddot{V}_{m}-2 \Omega \dot{W}_{m}-\Omega^{2} V_{m}\right] \\
& -\sum_{m=1}^{\infty} \rho A\left[\ddot{V}_{m}-2 \Omega \dot{W}_{m}-\Omega^{2} V_{m}\right]\left(\frac{2 L}{n \pi}\right) \sin \left(\frac{n \pi}{2}\right) \\
& -\sum_{m=1}^{\infty} \rho A\left[\ddot{V}_{m}-2 \Omega \dot{W}_{m}-\Omega^{2} V_{m}\right]\left(\frac{2 L}{m \pi}\right) \sin \left(\frac{m \pi}{2}\right) \\
& +\frac{\rho A L}{2}\left[\ddot{V}_{n}-2 \Omega \dot{W}_{n}-\Omega^{2} V_{n}\right] \\
& +\sum_{m=1}^{\infty} M\left[\ddot{V}_{m}-2 \Omega \dot{W}_{m}-\Omega^{2} V_{m}\right] \\
& -\sum_{m=1}^{\infty} M\left[\ddot{V}_{m}-2 \Omega \dot{W}_{m}-\Omega^{2} V_{m}\right] \cos \left(\frac{n \pi}{2}\right) \\
& -\sum_{m=1}^{\infty} M\left[\ddot{V}_{m}-2 \Omega \dot{W}_{m}-\Omega^{2} V_{m}\right] \cos \left(\frac{m \pi}{2}\right) \\
& +\sum_{m=1}^{\infty} M\left[\ddot{V}_{m}-2 \Omega \dot{W}_{m}-\Omega^{2} V_{m}\right] \\
& \cos \left(\frac{m \pi}{2}\right) \cos \left(\frac{n \pi}{2}\right)=0,
\end{aligned}
$$

$$
\begin{aligned}
\frac{L}{2}\left[S\left(\frac{n \pi}{2 L}\right)^{4} W_{n}+P\left(\frac{n \pi}{2 L}\right)^{2} W_{n}+\rho I\left(\frac{n \pi}{2 L}\right)^{2} \ddot{W}_{n}\right] \\
\quad+\sum_{m=1}^{\infty} \rho A L\left[\ddot{W}_{m}+2 \Omega \dot{V}_{m}-\Omega^{2} W_{m}\right] \\
\quad-\sum_{m=1}^{\infty} \rho A\left[\ddot{W}_{m}+2 \Omega \dot{V}_{m}-\Omega^{2} W_{m}\right]\left(\frac{2 L}{n \pi}\right) \sin \left(\frac{n \pi}{2}\right) \\
\quad-\sum_{m=1}^{\infty} \rho A\left[\ddot{W}_{m}+2 \Omega \dot{V}_{m}-\Omega^{2} W_{m}\right]\left(\frac{2 L}{m \pi}\right) \sin \left(\frac{m \pi}{2}\right) \\
+\frac{\rho A L}{2}\left[\ddot{W}_{m}+2 \Omega \dot{V}_{m}-\Omega^{2} W_{m}\right] \\
+\sum_{m=1}^{\infty} M\left[\ddot{W}_{m}+2 \Omega \dot{V}_{m}-\Omega^{2} W_{m}\right] \\
\quad-\sum_{m=1}^{\infty} M\left[\ddot{W}_{n}+2 \Omega \dot{V}_{n}-\Omega^{2} W_{n}\right] \cos \left(\frac{n \pi}{2}\right) \\
\quad-\sum_{m=1}^{\infty} M\left[\ddot{W}_{m}+2 \Omega \dot{V}_{m}-\Omega^{2} W_{m}\right] \cos \left(\frac{m \pi}{2}\right) \\
+\sum_{m=1}^{\infty} M\left[\ddot{W}_{m}+2 \Omega \dot{V}_{m}-\Omega^{2} W_{m}\right] \\
+\cos \left(\frac{m \pi}{2}\right) \cos \left(\frac{n \pi}{2}\right)=0 . \\
\quad
\end{aligned}
$$

To calculate natural frequencies of the micro-drill rotor system, time-dependent functions of $V_{n}(t)$ and $W_{n}(t)$ are considered as follows:

$$
\left\{\begin{array}{c}
V_{n}(t) \\
W_{n}(t)
\end{array}\right\}=\left\{\begin{array}{c}
\tilde{V}_{n} \\
\tilde{W}_{n}
\end{array}\right\} e^{i \omega t}
$$

where $\tilde{V}_{n}$ and $\tilde{W}_{n}$ are constants, $\omega$ is angular frequency, and $i=\sqrt{-1}$ is a complex number.

Substituting Eq. (31) into Eqs. (29) and (30), the following set of equations can be obtained:

$$
\begin{gathered}
\frac{L}{2}\left[S\left(\frac{n \pi}{2 L}\right)^{4} \tilde{V}_{n}+P\left(\frac{n \pi}{2 L}\right)^{2} \tilde{V}_{n}-\rho I\left(\frac{n \pi}{2 L}\right)^{2} \omega^{2} \tilde{V}_{n}\right] \\
+\sum_{m=1}^{\infty} \rho A L\left[-\omega^{2} \tilde{V}_{m}-2 \Omega i \omega \tilde{W}_{m}-\Omega^{2} \tilde{V}_{m}\right] \\
\quad-\sum_{m=1}^{\infty} \rho A\left[-\omega^{2} \tilde{V}_{m}-2 \Omega i \omega \tilde{W}_{m}-\Omega^{2} \tilde{V}_{m}\right], \\
\left(\frac{2 L}{n \pi}\right) \sin \left(\frac{n \pi}{2}\right)
\end{gathered}
$$




$$
\begin{aligned}
& -\sum_{m=1}^{\infty} \rho A\left[-\omega^{2} \tilde{V}_{m}-2 \Omega i \omega \tilde{W}_{m}-\Omega^{2} \tilde{V}_{m}\right] \\
& \left(\frac{2 L}{m \pi}\right) \sin \left(\frac{m \pi}{2}\right) \\
& +\frac{\rho A L}{2}\left[-\omega^{2} \tilde{V}_{n}-2 \Omega i \omega \tilde{W}_{n}-\Omega^{2} \tilde{V}_{n}\right] \\
& +\sum_{m=1}^{\infty} M\left[-\omega^{2} \tilde{V}_{m}-2 \Omega i \omega \tilde{W}_{m}-\Omega^{2} \tilde{V}_{m}\right] \\
& -\sum_{m=1}^{\infty} M\left[-\omega^{2} \tilde{V}_{m}-2 \Omega i \omega \tilde{W}_{m}-\Omega^{2} \tilde{V}_{m}\right] \cos \left(\frac{n \pi}{2}\right) \\
& -\sum_{m=1}^{\infty} M\left[-\omega^{2} \tilde{V}_{m}-2 \Omega i \omega \tilde{W}_{m}-\Omega^{2} \tilde{V}_{m}\right] \cos \left(\frac{m \pi}{2}\right) \\
& +\sum_{m=1}^{\infty} M\left[-\omega^{2} \tilde{V}_{m}-2 \Omega i \omega \tilde{W}_{m}-\Omega^{2} \tilde{V}_{m}\right] \\
& \cos \left(\frac{m \pi}{2}\right) \cos \left(\frac{n \pi}{2}\right)=0, \\
& \frac{L}{2}\left[S\left(\frac{n \pi}{2 L}\right)^{4} \tilde{W}_{n}+P\left(\frac{n \pi}{2 L}\right)^{2} \tilde{W}_{n}-\rho I\left(\frac{n \pi}{2 L}\right)^{2} \omega^{2} \tilde{W}_{n}\right] \\
& +\sum_{m=1}^{\infty} \rho A L\left[-\omega^{2} \tilde{W}_{m}+2 \Omega i \omega \tilde{V}_{m}-\Omega^{2} \tilde{W}_{m}\right] \\
& -\sum_{m=1}^{\infty} \rho A\left[-\omega^{2} \tilde{W}_{m}+2 \Omega i \omega \tilde{V}_{m}-\Omega^{2} \tilde{W}_{m}\right] \\
& \left(\frac{2 L}{n \pi}\right) \sin \left(\frac{n \pi}{2}\right) \\
& -\sum_{m=1}^{\infty} \rho A\left[-\omega^{2} \tilde{W}_{m}+2 \Omega i \omega \tilde{V}_{m}-\Omega^{2} \tilde{W}_{m}\right] \\
& \left(\frac{2 L}{m \pi}\right) \sin \left(\frac{m \pi}{2}\right) \\
& +\frac{\rho A L}{2}\left[-\omega^{2} \tilde{W}_{n}+2 \Omega i \omega \tilde{V}_{n}-\Omega^{2} \tilde{W}_{n}\right] \\
& +\sum_{m=1}^{\infty} M\left[-\omega^{2} \tilde{W}_{m}+2 \Omega i \omega \tilde{V}_{m}-\Omega^{2} \tilde{W}_{m}\right]
\end{aligned}
$$

$$
\begin{aligned}
& -\sum_{m=1}^{\infty} M\left[-\omega^{2} \tilde{W}_{m}+2 \Omega i \omega \tilde{V}_{m}-\Omega^{2} \tilde{W}_{m}\right] \\
& \cos \left(\frac{n \pi}{2}\right)-\sum_{m=1}^{\infty} M\left[-\omega^{2} \tilde{W}_{m}+2 \Omega i \omega \tilde{V}_{m}-\Omega^{2} \tilde{W}_{m}\right] \\
& \cos \left(\frac{m \pi}{2}\right)+\sum_{m=1}^{\infty} M\left[-\omega^{2} \tilde{W}_{m}+2 \Omega i \omega \tilde{V}_{m}-\Omega^{2} \tilde{W}_{m}\right] \\
& \cos \left(\frac{m \pi}{2}\right) \cos \left(\frac{n \pi}{2}\right)=0 .
\end{aligned}
$$

For an approximation, to calculate the first two natural frequencies of the system, in the above equations, the first two modes will be considered. For $n=1$ and $n=2$, Eqs. (32) and (33) can be written in the form of matrices as follows:

$$
\left[\begin{array}{ll}
A_{11} & A_{12} \\
A_{21} & A_{22}
\end{array}\right]\left\{\begin{array}{c}
\tilde{V}_{n} \\
\tilde{W}_{n}
\end{array}\right\}=\left\{\begin{array}{l}
0 \\
0
\end{array}\right\}
$$

where:

$$
\begin{aligned}
& A_{11}= A_{22}\left\{\left[\frac{S L}{2}\left(\frac{n \pi}{2 L}\right)^{4}+\frac{P L}{2}\left(\frac{n \pi}{2 L}\right)^{2}\right]\right. \\
&+\rho A L \Omega^{2}\left[-1.5+2\left(\frac{2}{n \pi}\right) \sin \left(\frac{n \pi}{2}\right)\right] \\
&\left.+M \Omega^{2}\left[-1+2 \cos \left(\frac{n \pi}{2}\right)-\cos ^{2}\left(\frac{n \pi}{2}\right)\right]\right\} \\
&+\left\{-\rho I\left[\frac{L}{2}\left(\frac{n \pi}{2 L}\right)^{2}\right]\right. \\
&+\rho A L\left[-1.5+\left(\frac{4}{n \pi}\right) \sin \left(\frac{n \pi}{2}\right)\right] \\
&\left.+M\left[-1+2 \cos \left(\frac{n \pi}{2}\right)-\cos ^{2}\left(\frac{n \pi}{2}\right)\right]\right\} \omega^{2},(35) \\
& A_{12}=-A_{21}=\left\{\rho A L\left[-3+4\left(\frac{2}{n \pi}\right) \sin \left(\frac{n \pi}{2}\right)\right]\right. \\
&\left.+M\left[-2+4 \cos \left(\frac{n \pi}{2}\right)-2 \cos ^{2}\left(\frac{n \pi}{2}\right)\right]\right\} i \Omega \omega . \\
&(36)
\end{aligned}
$$

To have a non-zero solution for $\tilde{V}_{n}$ and $\tilde{W}_{n}$ in Eq. (34), the determinant of the coefficient matrix should be equal to zero. Therefore, the result can be written as follows:

$$
A_{11} A_{22}-A_{12} A_{21}=0 .
$$

Substituting Eqs. (35) and (36) into Eq. (37), for simplification, the obtained result can be determined as follows:

$$
\gamma_{1}^{2} \omega^{4}-\gamma_{2} \omega^{2}+\gamma_{3}^{2}=0
$$


where:

$$
\begin{aligned}
\gamma_{1}= & \rho I\left[-\frac{L}{2}\left(\frac{n \pi}{2 L}\right)^{2}\right] \\
& +\rho A L\left[-1.5+\left(\frac{4}{n \pi}\right) \sin \left(\frac{n \pi}{2}\right)\right] \\
& +M\left[-1+2 \cos \left(\frac{n \pi}{2}\right)-\cos ^{2}\left(\frac{n \pi}{2}\right)\right], \\
\gamma_{2}= & D_{3}^{2} \Omega^{2}-2 \gamma_{1} \gamma_{3}, \\
\gamma_{3}= & D_{1}+D_{2} \Omega^{2},
\end{aligned}
$$

where:

$$
\begin{aligned}
D_{1}= & {\left[\frac{S L}{2}\left(\frac{n \pi}{2 L}\right)^{4}+\frac{P L}{2}\left(\frac{n \pi}{2 L}\right)^{2}\right] } \\
D_{2}= & \rho A L\left[-1.5+2\left(\frac{2}{n \pi}\right) \sin \left(\frac{n \pi}{2}\right)\right] \\
& +M\left[-1+2 \cos \left(\frac{n \pi}{2}\right)-\cos ^{2}\left(\frac{n \pi}{2}\right)\right], \\
D_{3}= & \rho A L\left[-3+4\left(\frac{2}{n \pi}\right) \sin \left(\frac{n \pi}{2}\right)\right] \\
& +M\left[-2+4 \cos \left(\frac{n \pi}{2}\right)-2 \cos ^{2}\left(\frac{n \pi}{2}\right)\right] .
\end{aligned}
$$

From Eq. (38), two natural frequencies for each mode $n$ of the system, which correspond to forward whirling motion $\left(\omega_{n}^{F W}\right)$ and backward whirling motion $\left(\omega_{n}^{B W}\right)$, can be obtained as follows (for $n=1,2$ ):

$$
\begin{aligned}
& \omega_{n}^{F W}=+\left[\frac{\gamma_{2}+2 \gamma_{1} \gamma_{3}}{\left(2 \gamma_{1}\right)^{2}}\right]^{\frac{1}{2}}+\left[\frac{\gamma_{2}-2 \gamma_{1} \gamma_{3}}{\left(2 \gamma_{1}\right)^{2}}\right]^{\frac{1}{2}}, \\
& \omega_{n}^{B W}=-\left[\frac{\gamma_{2}+2 \gamma_{1} \gamma_{3}}{\left(2 \gamma_{1}\right)^{2}}\right]^{\frac{1}{2}}+\left[\frac{\gamma_{2}-2 \gamma_{1} \gamma_{3}}{\left(2 \gamma_{1}\right)^{2}}\right]^{\frac{1}{2}} .
\end{aligned}
$$

Substituting $\gamma_{i}$ from Eq. (39) into Eq. (41), for simplification, the obtained result can be written as follows (for $n=1,2$ ):

$$
\begin{aligned}
\omega_{n}^{F W}= & +\left[\frac{D_{3}^{2}}{\left(2 \gamma_{1}\right)^{2}}\right]^{\frac{1}{2}} \Omega \\
& +\left[\frac{\left(D_{3}^{2}-4 \gamma_{1} D_{2}\right) \Omega^{2}-4 \gamma_{1} D_{1}}{\left(2 \gamma_{1}\right)^{2}}\right]^{\frac{1}{2}}, \\
\omega_{n}^{B W}= & -\left[\frac{D_{3}^{2}}{\left(2 \gamma_{1}\right)^{2}}\right]^{\frac{1}{2}} \Omega \\
& +\left[\frac{\left(D_{3}^{2}-4 \gamma_{1} D_{2}\right) \Omega^{2}-4 \gamma_{1} D_{1}}{\left(2 \gamma_{1}\right)^{2}}\right]^{\frac{1}{2}} .
\end{aligned}
$$

If the rotational speed of the micro-drill rotor $\Omega$ surpasses a specific value, which is called threshold of instability speeds, we will not have a real value for the natural frequencies, i.e., the system becomes unstable. According to Eq. (42), threshold of instability speeds of the system can be obtained as follows:

$$
\Omega_{n}^{t h}=\left[\frac{4 \gamma_{1} D_{1}}{D_{3}^{2}-4 \gamma_{1} D_{2}}\right]^{\frac{1}{2}}, \quad \text { for } n=1,2,
$$

where the coefficients $\gamma_{1}$ and $D_{i}(i=1,2,3)$ have been given in Eqs. (39) and (40), respectively.

\section{Validation}

The governing equations (Eqs. (21) and (22)) for lateral vibration of the micro-drill system were derived analytically based on the MCST. In the special case of $\frac{l}{R} \approx 0$, for a macro system, the governing equations are simplified as:

$$
\begin{gathered}
E I \frac{\partial^{4} v}{\partial x^{4}}-P \frac{\partial^{2} v}{\partial x^{2}}+[\rho A+M \delta(x-L)] \\
\left(\ddot{v}+2 \Omega \dot{w}-\Omega^{2} v\right)-\rho I \frac{\partial^{2} \ddot{v}}{\partial x^{2}}=0, \\
E I \frac{\partial^{4} w}{\partial x^{4}}-P \frac{\partial^{2} W}{\partial x^{2}}+[\rho A+M \delta(x-L)] \\
\left(\ddot{w}+2 \Omega \dot{v}-\Omega^{2} w\right)-\rho I \frac{\partial^{2} \ddot{w}}{\partial x^{2}}=0 .
\end{gathered}
$$

Eqs. (44) and (45) are suitably compatible with the ones presented in $[51,55]$. Also, for the case of $\frac{l}{R} \approx 0$ and $\Omega=0$ (non-rotating drill), the governing equation (Eq. (22)) is simplified as:

$$
E I \frac{\partial^{4} w}{\partial x^{4}}-P \frac{\partial^{2} W}{\partial x^{2}}+[\rho A+M \delta(x-L)] \ddot{w}-\rho I \frac{\partial^{2} \ddot{w}}{\partial x^{2}}=0 .
$$

The above equation is the typical formulation for the lateral vibration of the Euler-Bernoulli beam comprising an attached point mass, $M$, at the free end under constant axial load, $P$. Moreover, according to Eq. (42), for the non-rotating drill $(\Omega=0)$ and buckling case, the first natural frequency has to be zero, that is:

$$
D_{1}=\left[\frac{S L}{2}\left(\frac{\pi}{2 L}\right)^{4}+\frac{P L}{2}\left(\frac{\pi}{2 L}\right)^{2}\right]=0 .
$$

Thus, the critical axial load $P_{c r}$ in the buckling case can be calculated from Eq. (47) as follows:

$$
P_{c r}=-S\left(\frac{\pi}{2 L}\right)^{2},
$$


where:

$$
S=E I\left[1+\frac{2}{1+\vartheta}\left(\frac{l}{R}\right)^{2}\right]
$$

In the macro system, the buckling load can be calculated from Eq. (49) for $\frac{l}{R} \approx 0$ as follows:

$$
P_{c r}=-\frac{E I \pi^{2}}{(2 L)^{2}} \text {. }
$$

The obtained buckling load is same as that of the classical solution to a cantilever beam with the clamped-free boundary conditions.

\section{Numerical results and discussion}

In this section, the effects of size on the dynamic behavior of a micro-drill rotor subjected to an axial load are studied and some numerical results are presented.

For numerical analyses, with regard to the mechanical properties and geometrical data, Young's modulus, $E=1.44 \mathrm{GPa}$, Poison's ratio, $\vartheta=0.38$, rotor density, $\rho=7800 \mathrm{~kg} / \mathrm{m}^{3}$, rotor radius, $R=20 \mu \mathrm{m}$, rotor length, $L=20 R \mu \mathrm{m}$ [50], and concentrated mass, $M=2.091 \times 10^{-9} \mathrm{~kg}$, are considered. The first natural frequencies for both forward and backward (FW and BW) whirling motions versus rotational speed of rotor, $\Omega$, are depicted in Figure 3 for different values of dimensionless material length scale parameter, $l / R$, concentrated mass of $M=2.91 \times 10^{-9} \mathrm{~kg}$, and axial load of $P=0 \mathrm{~N}$. The obtained results in Figure 3 show that the first natural frequencies of the system for both forward and backward whirling motions will increase with any increase in dimensionless material length scale parameter, $l / R$. The above behavior in the second natural frequency for both forward and backward whirling motions versus rotational speed of rotor, $\Omega$, can be seen in Figure 4 .

Also, the first natural frequency for both forward and backward whirling motions in the micro-drill rotor system versus axial load, $P$, is presented in Figure 5 for various values of dimensionless material length scale parameter, $l / R$, rotational speed of $\Omega=10000 \mathrm{rpm}$, and $l / R=20$. The results in Figure 5 show that the first natural frequency of the system will decrease when compressive axial load, $P$, increases. It should be noted that the negative value of axial load demonstrates that it is in the compressive case. Also, according to Figure 5, the numerical results show that the first natural frequency of the system will approach zero with increase in the compressive axial load $P$, which is called the buckling load for the micro-cantilevered rotor. In this case study, buckling load (compressive axial force) of the micro-drill rotor system is calculated $-2.80 \times 10^{-3},-3.80 \times 10^{-3}$, and $-6.80 \times 10^{-3} \mathrm{~N}$ when $l / R$ is equal to $0.0,0.5$, and 1.0 , respectively.

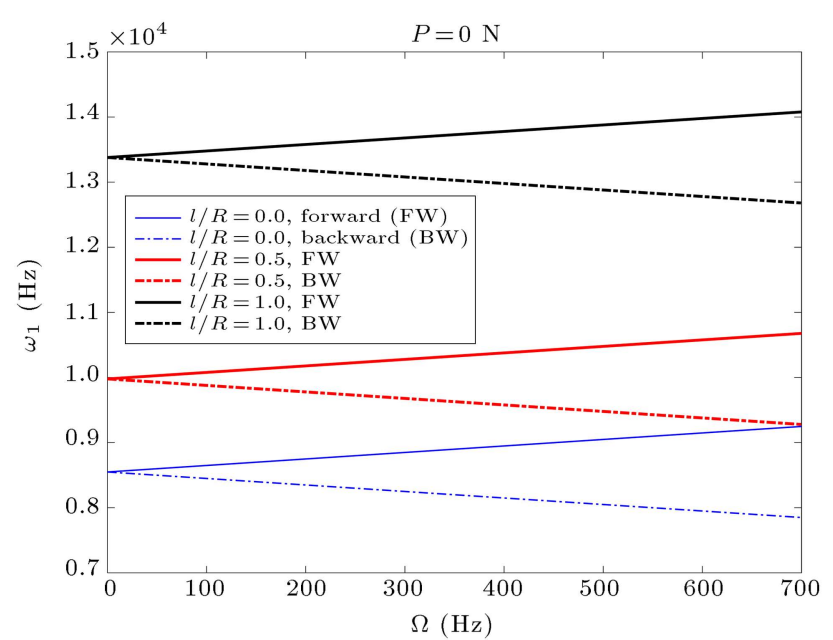

Figure 3. Variation of the first natural frequency of the micro-drill rotor system versus rotational speed of the rotor for different values of $l / R$ parameter.

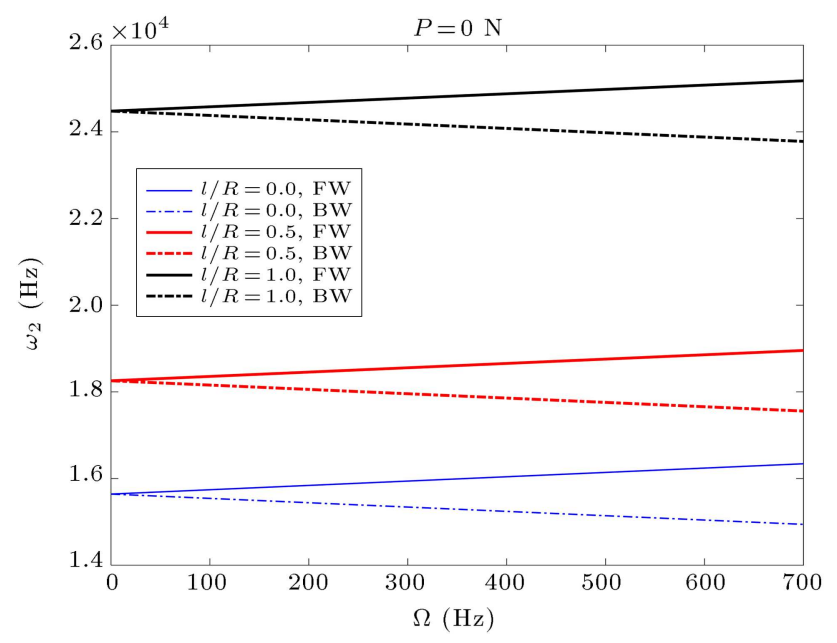

Figure 4. Variation of the second natural frequency of the micro-drill rotor system versus rotational speed of the rotor for different values of $l / R$ parameter.

Variations of threshold of instability speeds $\Omega_{1}^{t h}$ and $\omega_{2}^{\text {th }}$ of the micro-drill rotor system versus dimensionless material length scale parameter, $l / R$, for different values of the rotor length are presented in Figures 6 and 7 , respectively, when compressive axial load, $P$, is $5 \times 10^{-4} \mathrm{~N}$. The results show that the values of $\Omega_{1}^{t h}$ and $\Omega_{2}^{t h}$ rise as $L / R$ decreases and $l / R$ increases. The first natural frequency for the forward whirling motion of the micro-drill rotor system versus dimensionless material length scale parameter $l / R$ is provided in Figure 8 for different values of the rotor length at $\Omega=10000 \mathrm{rpm}$ and axial load of $P=$ $-5 \times 10^{-4} \mathrm{~N}$. The results in Figure 8 also show that the first natural frequency for forward whirling motion of the micro-drill rotor system increases when the rotor length decreases and the dimensionless material length scale parameter, $l / R$, increases. 


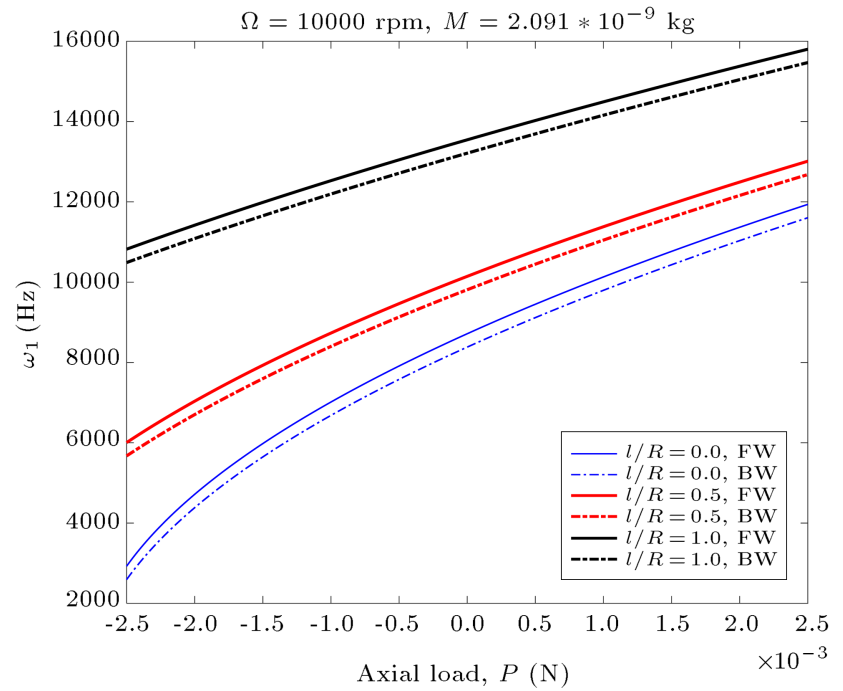

Figure 5. Variation of the first natural frequency of the micro-drill rotor system versus axial load, $P$, for different values of $l / R$ parameter at $\Omega=10000 \mathrm{rpm}$.

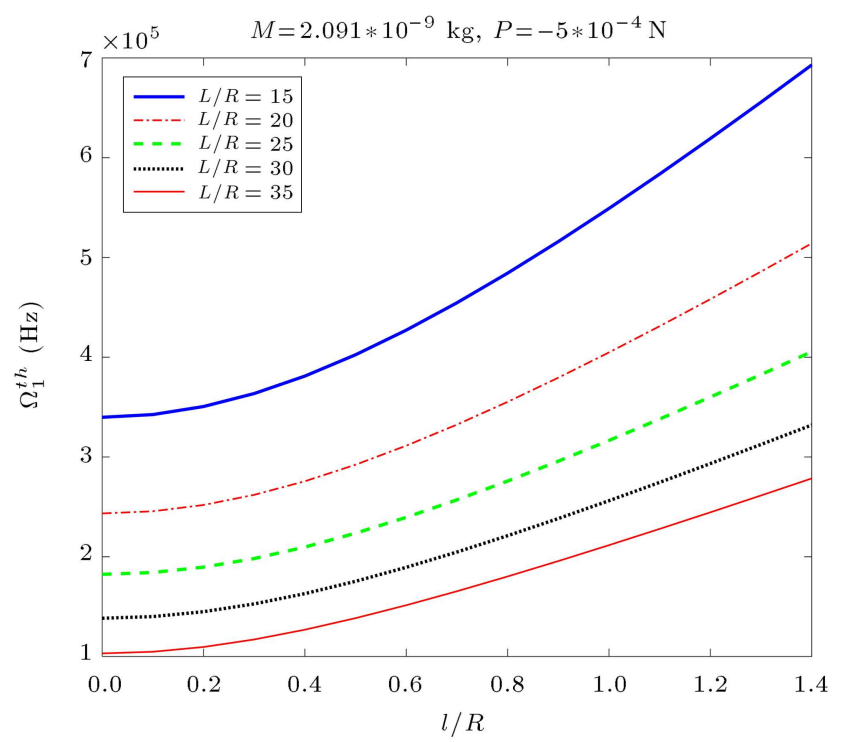

Figure 6. Variation of the first mode threshold of instability speed of the micro-drill rotor system versus $l / R$ parameter for different values of the rotor length at $P=-5 \times 10^{-4} \mathrm{~N}$.

Variation of the first natural frequency for the forward whirling motion of the micro-drill rotor system versus concentrated mass, $M$, with different values of dimensionless material length scale parameter, $l / R$, at $\Omega=10000 \mathrm{rpm}$ is investigated in Figure 9. The numerical results in Figure 9 show that the first natural frequency for forward whirling motion of the microdrill rotor system decreases when $l / R$ decreases and $M$ increases. Finally, variation of buckling load (compressive force) versus dimensionless material length scale parameter, $l / R$, is depicted in Figure 10 for different values of the rotor length. The results in Figure 10

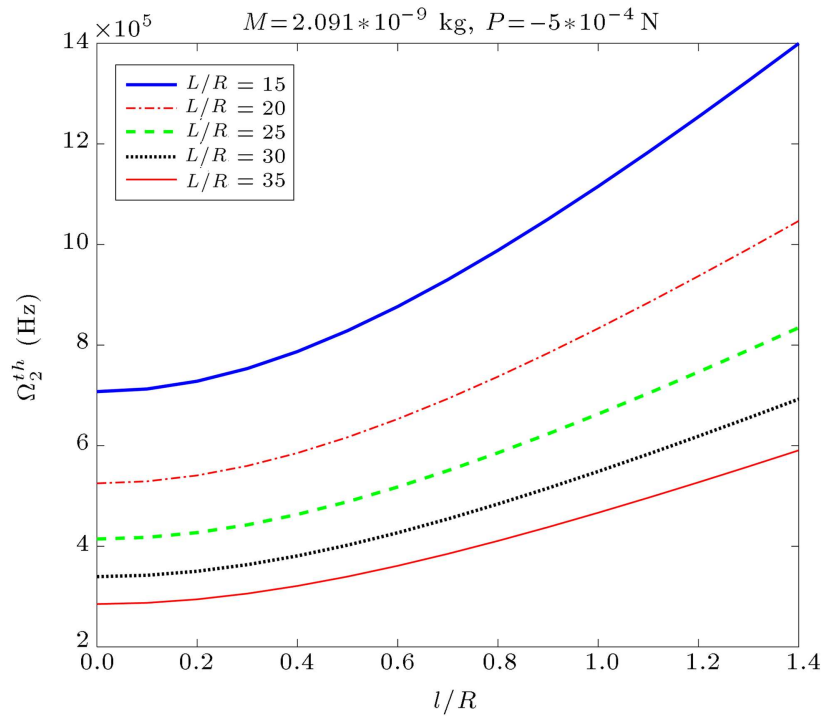

Figure 7. Variation of the second-mode threshold of instability speeds of the micro-drill rotor system versus $l / R$ parameter for different values of the rotor length at $P=-5 \times 10^{-4} \mathrm{~N}$.

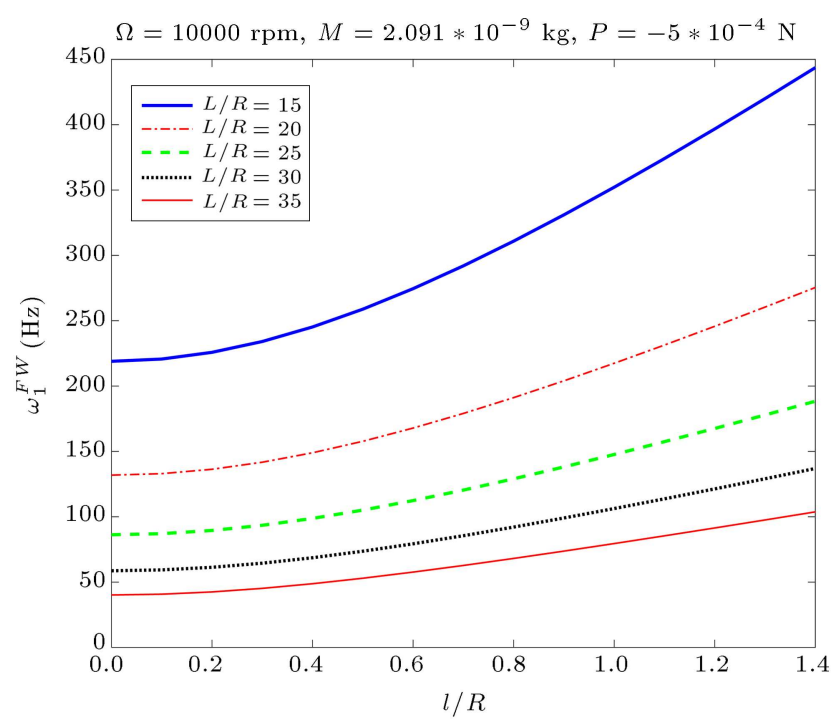

Figure 8. Variation of the first natural frequency for forward whirling motion of the micro-drill rotor system versus $l / R$ parameter for different values of the rotor length at $\Omega=10000 \mathrm{rpm}$ and $P=-5 \times 10^{-4} \mathrm{~N}$.

show that buckling load of the micro-drill rotor system will increase when the rotor length decreases and the dimensionless material length scale parameter, $l / R$, increases.

General review of the results in Figures 3 to 9 for the size effect on dynamic behavior of the system shows that when the rotor radius, $R$, comes close to the material length scale parameter, $l$, in micro-scale, the first and second natural frequencies of the system increase by approximately 52 to 66 percent at $l / R=20$ and $P=0 \mathrm{~N}$, depending on rotational speed, $\Omega$. 


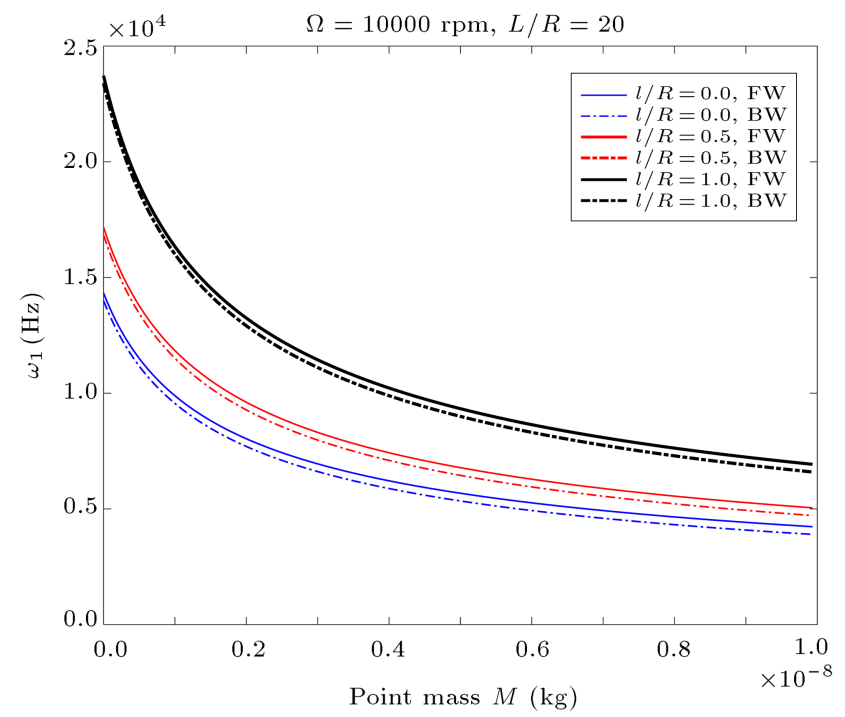

Figure 9. Variation of the first natural frequency for forward whirling motion of the micro-drill rotor system versus concentrated mass, $M$, for different values of $l / R$ parameter at $\Omega=10000 \mathrm{rpm}$.

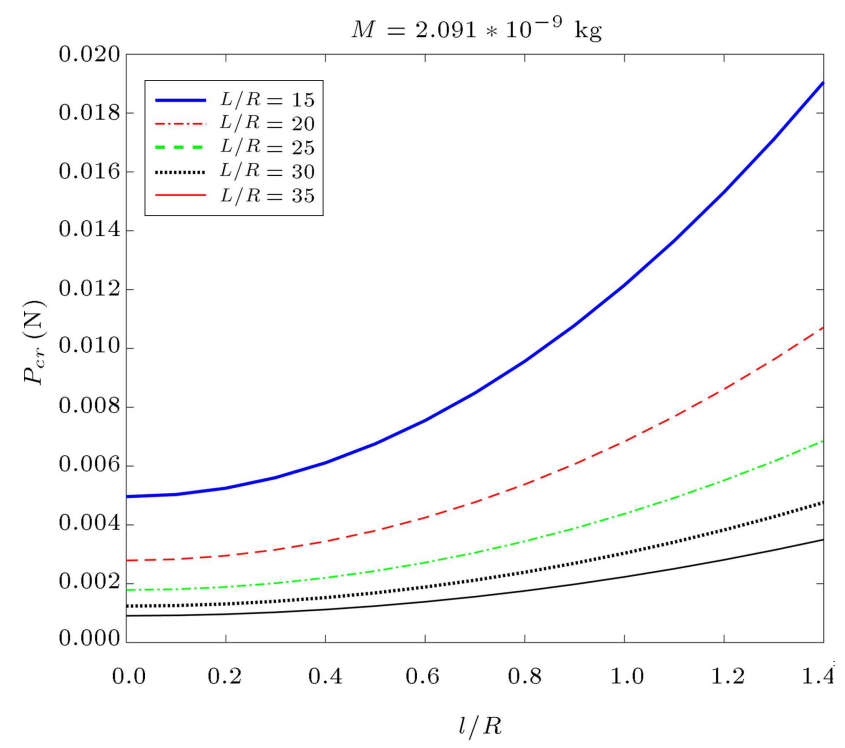

Figure 10. Variation of buckling load (compressive force) versus $l / R$ parameter.

\section{Summary and Conclusion}

A micro-cantilever rotor subjected to an axial load with a concentrated mass attached to the free end was considered for modeling of a micro-drill system, which could be used in the future applications in medical industries. The dynamic behavior of a microcantilever rotor system subjected to an axial load with a concentrated mass attached to the free end of the micro-rotor was studied. To capture size effects as well as to predict the dynamic behavior of the system, the governing equations of motion were derived based on the non-classical theory of MCST and Hamilton's principle. The Assumed Modes method was employed to transform the partial differential equations into a set of infinite ordinary differential equations. The first and second natural frequencies of the system for both forward and backward whirling motions were obtained by considering the first two modes.

Numerical results of the natural frequencies and the threshold of instability speeds of the system with respect to different values of system parameters were presented. The results showed that material length scale parameter values, axial load values, and concentrated mass values had significant effects on vibration characteristics of the micro-drill rotor system.

\section{References}

1. Jeon, B.S., Park, K.J., Jin Song, S., Joo, Y.C., and Min, K.D. "Design, fabrication, and testing of a MEMS microturbine", J. Mech. Sci. Technol., 19(2), pp. 682691 (2005).

2. Chou, S.K., Yang, W.M., Chua, K.J., Li, J., and Zhang, K.L. "Development of micro power generatorsa review", Appl. Energ., 88(1), pp. 1-16 (2011).

3. Iizuka, A., Takato, M., Kaneko, M., Nishi, T., Saito, K., and Uchikoba, F. "Millimeter scale MEMS air turbine generator by winding wire and multilayer magnetic ceramic circuit", Modern Mech. Eng., 2(02), pp. 41-46 (2012).

4. Schubert, D. "Mems-concept using micro turbines for satellite power supply", Solar Power In Tech, pp. 195210 (2012).

5. Pandey, R.K. and Panda, S.S. "Drilling of bone: a comprehensive review", J. Clinical Orthop. Trauma, 4(1), pp. 15-30 (2013).

6. Rysava, Z., Bruschi, S., Carmignato, S., Medeossi, F., Savio, E., and Zanini, F. "Micro-drilling and threading of the Ti6Al4V titanium alloy produced through additive manufacturing", Procedia CIRP, 46, pp. 583-586 (2016).

7. Zhang, W.M. and Meng, G. "Stability, bifurcation and chaos of a high-speed rub-impact rotor system in MEMS", Sens. Actuators A Phys., 127(1), pp. 163-178 (2006).

8. Meng, G., Zhang, W.M., Huang, H., Li, H.G., and Chen, D.I. "Micro-rotor dynamics for micro-electromechanical systems (MEMS)", Chaos Solitons Fractals, 40(2), pp. 538-562 (2009).

9. Fleck, N.A., Muller, G.M., Ashby, M.F., and Hutchinson, J.W. "Strain gradient plasticity: theory and experiment", Acta Metall. Mater., 42(2), pp. 475-487 (1994).

10. Stolken, J.S. and Evans, A.G. "A microbend test method for measuring the plasticity length scale", Acta Materialia, 46(14), pp. 5109-5115 (1998).

11. Lam, D.C. and Chong, A.C.M. "Indentation model and strain gradient plasticity law for glassy polymers", J. Mater. Res., 14(09), pp. 3784-3788 (1999). 
12. Chong, A.C.M. and Lam, D.C. "Strain gradient plasticity effect in indentation hardness of polymers", $J$. Mater. Res., 14(10), pp. 4103-4110 (1999).

13. Lam, D.C., Yang, F., Chong, A.C.M., Wang, J., and Tong, P. "Experiments and theory in strain gradient elasticity", J. Mech. Phys. Solids, 51(8), pp. 1477-1508 (2003).

14. Mindlin, R.D. and Tiersten, H.F. "Effects of couplestresses in linear elasticity", Archive for Rational Mechanics and Analysis, Arch. Rational Mech. Anal., 11(1), pp. 415-448 (1962).

15. Eringen, A.C. "Nonlocal polar elastic continua", Int. J. Eng. Sci., 10(1), pp. 1-16 (1972).

16. Yang, F., Chong, A.C.M., Lam, D.C., and Tong, P. "Couple stress based strain gradient theory for elasticity", Int. J. Solids Struct., 39(10), pp. 2731-2743 (2002).

17. Gurtin, M.E. and Murdoch, A.I. "A continuum theory of elastic material surfaces", Arch. Rational Mech. Anal., 57(4), pp. 291-323 (1975).

18. Gurtin, M.E. and Murdoch, A.I. "Surface stress in solids", Int. J. Solids Struct., 14(6), pp. 431-440 (1978).

19. Abderezaei, J. and Moghimi Zand, M. "Transient behavior of electrostatically-actuated micro systems considering squeeze film damping and mechanical shock", Scientia Iranica, 24(6), pp. 2887-2894 (2017). DOI: $10.24200 /$ sci. 2017.4245

20. Jabbarian, S. and Ahmadian, M. "Free vibration analysis of functionally graded stiffened micro-cylinder based on the modified couple stress theory", Scientia Iranica, Transactions B, Mechanical Engineering, 25(5), pp. 2598-2615 (2018).

21. Rahi, A. "Lateral vibrations of a microrotating shaft-disk system subjected to an axial load based on the modified strain gradient theory", Mechanics of Advanced Materials and Structures (2018). DOI: 10.1080/15376494.2018.1444223

22. Shenas, A.G., Ziaee, S., and Malekzadeh, P. "Vibrational behavior of rotating pre-twisted functionally graded microbeams in thermal environment", Compos. Struct., 157, pp. 222-235 (2016).

23. Shenas, A.G., Malekzadeh, P., and Mohebpour, S. "Vibrational behavior of variable section functionally graded microbeams carrying microparticles in thermal environment", Thin-Walled Struct., 108, pp. 122-137 (2016).

24. Mindlin, R.D. "Second gradient of strain and surfacetension in linear elasticity", Int. J. Solids Struct., 1(4), pp. 417-438 (1965).

25. Mindlin, R.D. and Eshel, N.N. "On first straingradient theories in linear elasticity", Int. J. Solids Struct., 4(1), pp. 109-124 (1968).

26. Fleck, N.A. and Hutchinson, J.W. "A phenomenological theory for strain gradient effects in plasticity", $J$. Mech. Phys. Solids, 41(12), pp. 1825-1857 (1993).
27. Lam, D.C., Yang, F., Chong, A.C.M., Wang, J., and Tong, P. "Experiments and theory in strain gradient elasticity", J. Mech. Phys. Solids, 51(8), pp. 1477-1508 (2003).

28. Park, S.K. and Gao, X.L. "Bernoulli-Euler beam model based on a modified couple stress theory", $J$. Micromech. Microeng., 16(11), pp. 2355-2359 (2006).

29. Ma, H.M., Gao, X.L., and Reddy, J.N. "A microstructure-dependent Timoshenko beam model based on a modified couple stress theory", J. Mech. Phys. Solids, 56(12), pp. 3379-3391 (2008).

30. Asghari, M., Kahrobaiyan, M.H., Rahaeifard, M., and Ahmadian, M.T. "Investigation of the size effects in Timoshenko beams based on the couple stress theory", Arch. Appl. Mech., 81(7), pp. 863-874 (2011).

31. Ke, L.L., Wang, Y.S., Yang, J., and Kitipornchai, S. "Nonlinear free vibration of size-dependent functionally graded microbeams", Int J Eng Sci., 50(1), pp. 256-267 (2012).

32. Liang, L.N., Ke, L.L., Wang, Y.S., Yang, J., and Kitipornchai, S. "Flexural vibration of an atomic force microscope cantilever based on modified couple stress theory", Int. J. Struct. Stab. Dyn., 15(07), p. 1540025 (2015).

33. Ghiasi, E.K. "Application of modified couple stress theory to study dynamic characteristics of electrostatically actuated micro-beams resting upon squeeze-film damping under mechanical shock", Int. J. Adv. Mater. Sci. Eng., 6(1), pp. 1-15 (2016).

34. Dai, H.L., Wang, Y.K., and Wang, L. "Nonlinear dynamics of cantilevered microbeams based on modified couple stress theory", Int. J. Eng. Sci., 94, pp. 103-112 (2015).

35. Simsek, M. "Size dependent nonlinear free vibration of an axially functionally graded (AFG) microbeam using He's variational method", Compos. Struct., 131, pp. 207-214 (2015).

36. Shafiei, N., Mousavi, A., and Ghadiri, M. "Vibration behavior of a rotating non-uniform FG microbeam based on the modified couple stress theory and GDQEM", Compos. Struct., 149, pp. 157-169 (2016).

37. Tsiatas, G.C. "A new Kirchhoff plate model based on a modified couple stress theory", Int. J. Solids Struct., 46(13), pp. 2757-2764 (2009).

38. Ansari, R., Faghih Shojaei, M., Mohammadi, V., Gholami, R., and Darabi, M.A. "Size-dependent vibrations of post-buckled functionally graded Mindlin rectangular microplates", Lat. Am. J. Solids Stru., 11(13), pp. 2351-2378 (2014).

39. Wang, K.F., Kitamura, T., and Wang, B. "Nonlinear pull-in instability and free vibration of micro/nanoscale plates with surface energy-a modified couple stress theory model", Int. J. Mech. Sci., 99, pp. 288-296 (2015).

40. Askari, A.R. and Tahani, M. "Size-dependent dynamic pull-in analysis of geometric non-linear micro-plates 
based on the modified couple stress theory", Physica E: Low-dimens. Syst. Nanostruct., 86, pp. 262-274 (2017).

41. Alinaghizadeh, F., Shariati, M., and Fish, J. "Bending analysis of size-dependent functionally graded annular sector microplates based on the modified couple stress theory", Appl. Math. Model., 44, pp. 540-556 (2017).

42. He, D., Yang, W., and Chen, W. "A size-dependent composite laminated skew plate model based on a new modified couple stress theory", Acta Mech. Solida Sin., 30(1), pp. 75-86 (2017).

43. Simsek, M. and Aydın, M. "Size-dependent forced vibration of an imperfect functionally graded (FG) microplate with porosities subjected to a moving load using the modified couple stress theory", Compos. Struct., 160, pp. 408-421 (2017).

44. Guo, J., Chen, J., and Pan, E. "Free vibration of threedimensional anisotropic layered composite nanoplates based on modified couple-stress theory", Physica E: Low-dimens. Syst. Nanostruct., 87, pp. 98-106 (2017).

45. Abadi, M.M. and Daneshmehr, A.R. "An investigation of modified couple stress theory in buckling analysis of micro composite laminated Euler-Bernoulli and Timoshenko beams", Int. J. Eng. Sci., 75, pp. 40-53 (2014).

46. Tang, M., Ni, Q., Wang, L., Luo, Y., and Wang, Y. "Nonlinear modeling and size-dependent vibration analysis of curved microtubes conveying fluid based on modified couple stress theory", Int. J. Eng. Sci., 84, pp. 1-10 (2014).

47. Hosseini-Hashemi, S., Sharifpour, F., and Ilkhani, M.R. "On the free vibrations of size-dependent closed micro/nano-spherical shell based on the modified couple stress theory", Int. J. Mech. Sci., 115, pp. 501-515 (2016).

48. Vatankhah, R. and Kahrobaiyan, M.H. "Investigation of size-dependency in free-vibration of microresonators based on the strain gradient theory", Lat. Am. J. Solids Stru., 13(3), pp. 498-515 (2016).
49. Dehrouyeh-Semnani, A.M., BehboodiJouybari, M., and Dehrouyeh, M. "On size-dependent lead-lag vibration of rotating microcantilevers", Int J Eng Sci., 101, pp. 50-63 (2016).

50. Hashemi, M. and Asghari, M. "Investigation of the small-scale effects on the three-dimensional flexural vibration characteristics of a basic model for microengines", Acta Mech., 226(9), pp. 3085-3096 (2015).

51. Yim, K.B. and Yim, J. "Dynamic behavior of overhung rotors subjected to axial forces", Int. J. Precis. Eng. Man., 13(9), pp. 1575-1580 (2012).

52. Tiaki, M.M., Hosseini, S.A.A., and Zamanian, M. "Nonlinear forced vibrations analysis of overhung rotors with unbalanced disk", Arch. Appl. Mech., 86(5), pp. $797-817$ (2016).

53. Dental Simulation, Sweden (2017). http://www. sensegraphics.com/simulation/dentalsimulation/

54. EureKAlert AAAS, The American Association for the Advancement of Science (AAAS), https:// www.eurekalert.org/pub_releases/2015-12/uosasf120315.php (2017).

55. Chang, C.O. and Cheng, J.W. "Non-linear dynamics and instability of a rotating shaft-disk system", $J$. Sound Vib., 160(3), pp. 433-454 (1993).

\section{Biography}

Abbas Rahi is an Assistant Professor in the Faculty of Mechanical and Energy Engineering at Shahid Beheshti University, Tehran, Iran. He received his $\mathrm{PhD}$ in Mechanical Engineering from AmirKabir University of Technology, Tehran, Iran. His main research activities include vibration analysis of structural elements and machinery using theoretical and experimental methods. Rotor dynamics, condition monitoring of the systems, and dynamic and vibration analysis of the microsystems are his other research interests. 